\title{
Adaptive Control for Stochastic Systems with Unknown Parameters and Input Delay
}

\author{
Suping $\mathrm{Cao}^{1}$ and $\mathrm{Xizhen} \mathrm{Hu}^{2}$ \\ ${ }^{1}$ School of Automation, Huazhong University of Science and Technology, Wuhan \\ 430074, China \\ ${ }^{2}$ School of Mathematics and Statistics, Wuhan University, Wuhan 430072, China \\ E-mail: cao.suping@yahoo.com
}

\begin{abstract}
The adaptive dual control problem of a single-in single-out (SISO) stochastic system with unknown parameters and input delay is studied in this paper. The unknown parameters are assumed to be constant but belong to a known finite set. For each group of possible model parameters, a Kalman filter is used to estimate the system state online and the posterior probability of this model can also be calculated accordingly. The optimal control of each model is designed, based on an M steps ahead loss function. The final dual controller is obtained by a weighted sum of all possible optimal control outputs considering the posterior probabilities of each model. Numerical simulations were performed to verify the effectiveness of the proposed controller.
\end{abstract}

Keywords: Adaptive dual control; Uncertain systems; Input delay; Kalman filter

\section{Introduction}

In recent literatures, a plenty of practical control problems have been known as dual control. In the 60's of last century, Feldbaum set up the theoretical foundation of the dual control problem for stochastic uncertain systems [1] [2]. The basic idea of dual control is identifying unknown system parameters through perturbing the control process while controlling it as well as possible. Therefore the controller needs to have dual features, which are known as 'caution' and 'probing'. As pointed out by Feldbaum, the optimal adaptive dual control can be obtained by applying the method of dynamic programming to solve a Bellman equation. However, the Bellman equation is difficult to solve except a few very simple examples [3] [4]. The difficulty in finding the optimal solution of dual adaptive control law led to the development of various simplified sub-optimal approaches in practical systems.

There are two main classes of sub-optimal adaptive dual control approaches, namely the implicit and explicit dual methods. The first ones try to solve the Bellman equation by using some approximated solutions of dynamic programming [5] [6]. In the second class, the optimization problem is reformulated by modifying cost functions [7] [8] [9]. Wittenmark proposed a controller using a one-step-ahead control objective considering the variance of the parameter-error in explicit form [4]. Maitelli presented a two-stage sub-optimal dual controller for a class of linear systems with unknown and randomly varying parameters [10]. In his method, the loss function which is minimized consists of the sum of output variances up to two steps ahead in time. Milito introduced a novel cost function which includes the variance of the innovations process [9]. A control law was then derived which has an explicit solution that allows for an easy implementation, and has dual properties. Li et. al studied a class of dual control problems where there exists a parameter uncertainty in the observation equation of the linear-quadratic Gaussian problem [11]. They obtained an analytical active dual control law by a variance minimization approach. In [12], they extended the approaches to a general class of discrete-time LQG problems with unknown parameters in both state and 
observation equations. However, to the best of our knowledge there are few researches considering the dual control problem of systems with input or state delay.

The input delay can be often encountered in many practical control systems, including the remote control or networked control systems (NCSs), the biological systems, etc. Ramesh et.al analyzed the dual effect existed in the state-based scheduling for NCSs [13]. Whereas, they din't discuss how to design a dual controller for a delay NSC. In [14], Maitelli et. al extended the design technique of sub-optimal dual controller proposed in [10] to a blood pressure measurement system, which can be modeled by a time-varying, discrete-time, singleinput-single-output (SISO) system with a generic time-delay. In their study, the time-varying parameters are modeled as a Gauss-Markov process.

In this paper, the dual control problem for another kind of discrete-time SISO systems with input delay is discussed. The system model is described by a delay difference equation with uncertain but constant parameters. Further, these uncertain constant parameters are assumed to belong to a finite set. The rest of this paper is organized as follows. Section 2 gives the formulation of control models and the definition of loss function. In section 3, the detailed design procedure of proposed dual controller is presented. Section 4 shows the simulation results and the paper is finally concluded in section 5 .

\section{Formulation}

In this section, the formulation of the general system model and the loss function is discussed. Let us consider the following SISO system with constant input delay $d$ :

$$
y(k)+a_{1} y(k-1)+\cdots+a_{m} y(k-m)=b_{1} u(k-d)+\cdots+b_{n} u(k-d-n+1)+e(k)
$$

where $y$ is the output and $u$ is the input. $\{e(k), k=1,2, \ldots\}$ is used to denote the white Gaussian noise sequence with zero mean and known variance $\sigma^{2} . \mathbf{I}^{0}$ is used to denote the initial information set including the probability distribution of initial output $y(0)$ and the statistics of random sequence $e(k)$. Accordingly, let us define the information set at time $k$ to be

$$
\mathbf{I}^{k}=\left\{u(0), \cdots, u(k-1), y(1), \cdots y(k), \mathbf{I}^{0}\right\}, \quad k=0,1, \cdots, N
$$

To simplify the notation, let us introduce a parameter vector $\boldsymbol{\theta}$ and a information vector $\varphi(k)$ satisfying

$$
\begin{gathered}
\boldsymbol{\theta}=\left[a_{1}, \cdots, a_{m}, b_{1}, \cdots, b_{n}\right]^{T} \in R^{m+n} \\
\boldsymbol{\varphi}(k)=[-y(k-1), \cdots,-y(k-m), u(k-d), \cdots, u(k-d-n+1)] \in R^{1 \times(m+n)}
\end{gathered}
$$

Then, the original SISO model (1) can be rewritten as

$$
y(k)=\boldsymbol{\varphi}(k) \boldsymbol{\theta}+e(k)
$$

All the parameters in $\boldsymbol{\theta}$ are assumed to be constant over the whole horizon but belong to a finite set. That is, we have

$$
\boldsymbol{\theta} \in \Omega=\left\{\boldsymbol{\theta}_{1}, \cdots, \boldsymbol{\theta}_{L}\right\}
$$

The a-prior probabilities of parameters are given as $q_{s}(0)=P\left(\boldsymbol{\theta}=\boldsymbol{\theta}_{s} \mid \mathbf{I}^{0}\right)$ with $s=1$, $2, \ldots, L$. Similar to [Maitelli1997], we would like to study a multistage suboptimal dual controller for uncertain SISO system (1) with input delay. The purpose of control is to minimize the variance of the output about a known reference sequence $y_{r}(k)$ during the next $N$ step of control. The cost function that should be optimized is given by

$$
V_{N}=E\left\{\frac{1}{N} \sum_{i=1}^{N}\left(y(k+i)-y_{r}(k+i)\right)^{2}\right\}
$$

where the expectation is with respect to all underlying random variables. The control problem is then reduced to finding the control sequence $u(k), \cdots, u(k+N-d)$ that minimize the performance index (7) subject to equation (5). Theoretically, the optimal 
solution of control could be obtained by solving a dynamic programming. However, practically it is difficult to solve dynamic programming problem online due to the expectation calculation and the curse of dimensionality. Thus, in this paper we propose a multistage suboptimal dual controller which minimizes the system output deviation from the reference value $M$ steps ahead at each time $k$. The loss function is then given by

$$
V_{M}{ }^{\prime}=E\left\{\frac{1}{M} \sum_{i=1}^{M}\left(y^{\prime}(k+i)-y_{r}(k+i)\right)^{2} \mid \mathbf{I}^{k}\right\}
$$

where $y^{\prime}(k+i)$ is the predicted output satisfying

$$
y^{\prime}(k+i)=\overline{\boldsymbol{\varphi}}(k+i) \boldsymbol{\theta}+e(k+i)
$$

The new information vector $\bar{\varphi}(k+i)$ is generated by replacing all future outputs in $\boldsymbol{\varphi}(k+i)$ by optimal predictor's values. That is, we have

$$
\begin{gathered}
\bar{\varphi}(k+i)=\left[-y_{a}^{p}(k+i-1), \cdots,-y_{a}^{p}(k+1),-y(k), \cdots,-y(k+i-m),\right. \\
u(k-d+i), \cdots, u(k-d-n+1+i)]
\end{gathered}
$$

where

$$
y_{a}^{p}(k+j)=\bar{\varphi}(k+j) \boldsymbol{\theta} .
$$

\section{Controller Design}

If assuming $\boldsymbol{\theta}=\boldsymbol{\theta}_{s}$, we can derive the following state equation of system (1):

$$
\begin{aligned}
& \mathbf{x}(k)=\mathbf{A}_{s} \mathbf{x}(k-1)+\mathbf{B}_{s} \mathbf{u}(k-1) \\
& z(k)=y(k)=\mathbf{H} \mathbf{x}(k)+e(k)
\end{aligned}
$$

where

$$
\begin{gathered}
\mathbf{x}(k-1)=[y(k-m), y(k-m+1), \cdots, y(k-1)]^{T} \in R^{m}, \\
\mathbf{H}=[0, \cdots, 0,1] \in R^{1 \times m}, \boldsymbol{\theta}_{s}=\left[a_{1, s}, \cdots, a_{m, s}, b_{1, s}, \cdots, b_{n, s}\right]^{T} \in R^{m+n}, s=1,2, \cdots, L, \\
\mathbf{A}_{s}=\left[\begin{array}{lllll}
0 & 1 & 0 & \cdots & 0 \\
0 & 0 & 1 & \cdots & 0 \\
0 & 0 & 0 & \cdots & 0 \\
\vdots & \vdots & \vdots & \ddots & \vdots \\
-a_{m, s} & -a_{m-1, s} & -a_{m-2, s} & \cdots & -a_{1, s}
\end{array}\right] \in R^{m \times m}, \mathbf{B}_{s}=\left[\begin{array}{lll}
0 & \cdots & 0 \\
\vdots & \vdots & \vdots \\
0 & \cdots & 0 \\
b_{1, s} & \cdots & b_{n, s}
\end{array}\right] \in R^{m \times n} .
\end{gathered}
$$

From state model (12), the state estimate at time $k$ can be obtained by normal Kalman filter:

$$
\begin{aligned}
& \text { Predictor: }\left\{\begin{array}{l}
\hat{\mathbf{x}}(k \mid k-1)=\mathbf{A}_{s} \hat{\mathbf{x}}(k-1 \mid k-1)+\mathbf{B}_{s} \mathbf{u}(k-1) \\
\mathbf{P}_{s}(k \mid k-1)=\mathbf{A}_{s} \mathbf{P}_{s}(k-1 \mid k-1) \mathbf{A}_{s}{ }^{T}
\end{array}\right. \\
& \text { Corrector: }\left\{\begin{array}{l}
\hat{\mathbf{x}}(k \mid k)=\hat{\mathbf{x}}(k \mid k-1)+\mathbf{F}_{k}(z(k)-\mathbf{H} \hat{\mathbf{x}}(k \mid k-1)) \\
\mathbf{F}_{k}=\mathbf{P}_{s}(k \mid k-1) \mathbf{H}^{T}\left(\mathbf{H P}_{s}(k \mid k-1) \mathbf{H}^{T}+\sigma^{2}\right)^{-1} \\
\mathbf{P}_{s}(k \mid k)=\mathbf{P}_{s}(k \mid k-1)-\mathbf{F}_{k} \mathbf{H P}_{s}(k \mid k-1)
\end{array}\right.
\end{aligned}
$$

with initial condition $\hat{\mathbf{x}}(0 \mid 0)=\mathbf{x}(0), \mathbf{P}_{s}(0 \mid 0)=\mathbf{P}(0)$.

According to the observations, the posterior probability of model $s$ at time $k$ can be calculated recursively as follows [15]: 


$$
q_{s}(k)=\frac{L_{s}(k)}{\sum_{j=1}^{L} L_{j}(k) q_{j}(k-1)} q_{s}(k-1)
$$

where

$$
\begin{aligned}
& L_{s}(k)=\operatorname{det}\left[\mathbf{P}_{y}\left(k, \boldsymbol{\theta}_{s}\right)\right]^{-1 / 2} \exp \left\{-\frac{1}{2} \tilde{y}^{T}\left(k, \boldsymbol{\theta}_{s}\right) \mathbf{P}_{y}^{-1}\left(k, \boldsymbol{\theta}_{s}\right) \tilde{y}\left(k, \boldsymbol{\theta}_{s}\right)\right\} \\
& \mathbf{P}_{y}\left(k, \boldsymbol{\theta}_{s}\right)=\mathbf{H} \mathbf{P}_{s}(k-1 \mid k-1) \mathbf{H}^{T}+\sigma^{2} \\
& \tilde{y}\left(k, \boldsymbol{\theta}_{s}\right)=y(k)-\mathbf{H} \hat{\mathbf{x}}_{s}(k-1 \mid k-1)
\end{aligned}
$$

From (8) and (9) let us define the corresponding loss function of the $s$-th mode as

$$
\begin{aligned}
& V_{M s}{ }^{\prime}=E\left\{\frac{1}{M} \sum_{i=1}^{M}\left(\overline{\boldsymbol{\varphi}}(k+i) \boldsymbol{\theta}_{s}+e(k+i)-y_{r}(k+i)\right)^{2} \mid \mathbf{I}^{k}\right\} \\
& =\frac{1}{M} \sum_{i=1}^{M}\left[\overline{\boldsymbol{\varphi}}(k+i) \boldsymbol{\theta}_{s} \boldsymbol{\theta}_{s}^{T} \overline{\boldsymbol{\varphi}}^{T}(k+i)+y_{r}{ }^{2}(k+i)-2 \overline{\boldsymbol{\varphi}}(k+i) \boldsymbol{\theta}_{s} y_{r}(k+i)+\sigma^{2}\right]
\end{aligned}
$$

Let us introduce row vectors described by

$$
\mathbf{I}_{i}=\underbrace{[0, \cdots, 0,1,0, \cdots, 0]}_{\text {ith position is } 1} \in R^{1 \times(\mathrm{m}+\mathrm{n})}
$$

Then the vector $\overline{\boldsymbol{\varphi}}(k+i)$ can be rewritten as

$$
\begin{aligned}
\overline{\boldsymbol{\varphi}}(k+i) & =-\mathbf{l}_{1} y_{a}^{P}(k+i-1)-\cdots-\mathbf{l}_{i-1} y_{a}^{P}(k+1) \\
& +\mathbf{l}_{m+1} u(k+i-d)+\cdots+\mathbf{l}_{m+i-d+1} u(k)+\tilde{\boldsymbol{\varphi}}(k+i)
\end{aligned}
$$

where $\tilde{\boldsymbol{\varphi}}(k+i)$ is the vector including all the known information at time $k$ and is given by

$$
\begin{aligned}
\tilde{\boldsymbol{\varphi}}(k+i)= & {[0, \cdots,-y(k), \cdots,-y(k+i-m),} \\
& 0, \cdots, u(k-1), \cdots, u(k-d-n+i+1)]
\end{aligned}
$$

Substituting (11) to (18), it follows that

$$
\begin{aligned}
\overline{\boldsymbol{\varphi}}(k+i) & =-\mathbf{l}_{1} \overline{\boldsymbol{\varphi}}(k+i-1) \theta_{s}-\cdots-\mathbf{l}_{i-1} \overline{\boldsymbol{\varphi}}(k+1) \theta_{s} \\
& +\mathbf{l}_{m+1} u(k+i-d)+\cdots+\mathbf{l}_{m+i-d+1} u(k)+\tilde{\boldsymbol{\varphi}}(k+i)
\end{aligned}
$$

By carrying out some mathematical manipulations, from (20) $\bar{\varphi}(k+i)$ can be represented as a function of current inputs, future inputs and a known information vector $\tilde{\varphi}^{*}(k+i)$ at time $k$

$$
\overline{\boldsymbol{\varphi}}(k+i)=\mathbf{l}_{m+1}^{i-d+1} u(k+i-d)+\cdots+\mathbf{l}_{m+i-d+1}^{1} u(k)+\tilde{\boldsymbol{\varphi}}^{*}(k+i)
$$

where

$$
\begin{aligned}
& \tilde{\boldsymbol{\varphi}}^{*}(k+i)=\tilde{\boldsymbol{\varphi}}(k+i)-\sum_{p=1}^{i-1} \mathbf{l}_{p} \tilde{\boldsymbol{\varphi}}^{*}(k+i-p) \boldsymbol{\boldsymbol { \theta } _ { s }}, \text { with } \tilde{\boldsymbol{\varphi}}^{*}(k+1)=\tilde{\boldsymbol{\varphi}}(k+1) \\
& \left\{\begin{array}{l}
\mathbf{l}_{m+i}^{j}=\mathbf{l}_{m+i}-\sum_{p=1}^{i-1} \mathbf{l}_{i-p} \mathbf{K}_{p}^{j} \\
\mathbf{K}_{p}^{j}=\mathbf{l}_{m+p} \boldsymbol{\theta}_{s}-\sum_{q=1}^{p-1} \mathbf{l}_{p-q} \boldsymbol{\theta}_{s} \mathbf{K}_{q}^{j}
\end{array}\right.
\end{aligned}
$$

Let us define a control vector as

$$
\mathbf{u}=[u(k+M-d), u(k+M-d-1), \cdots, u(k)]^{T} \in R^{M-d+1} .
$$

From (21) we have 


$$
\overline{\boldsymbol{\varphi}}(k+i)=\tilde{\boldsymbol{\varphi}}^{*}(k+i)+\mathbf{u}^{T}\left[\begin{array}{l}
0 \\
\vdots \\
0 \\
\mathbf{l}_{m+1}^{i-d+1} \\
\vdots \\
\mathbf{l}_{m+i-d+1}^{1}
\end{array}\right]
$$

Substituting (24) to (16), the loss function can then be represented in a quadratic form:

$$
V_{M s}^{\prime}=\frac{1}{M} \sum_{i=1}^{M}\left[\frac{1}{2} \mathbf{u}^{T} \mathbf{S}_{i} \mathbf{u}+\mathbf{u}^{T} \mathbf{T}_{i, s}+\mathbf{c}_{i, s}\right]=\frac{1}{M}\left[\frac{1}{2} \mathbf{u}^{T} \mathbf{S u}+\mathbf{u}^{T} \mathbf{T}_{s}+\mathbf{c}_{s}\right]
$$

where $\mathbf{S}_{i} \in R^{(M-d+1) \times(M-d+1)}$ is a symmetric, positive definite matrix with the elements given by

$$
\mathbf{S}_{i}(h, j)=2 \cdot \mathbf{l}_{m+h+i-M}^{M-h-d+2}\left(\mathbf{l}_{m+j+i-M}^{M-j-d+2}\right)^{T}, \quad i=1, \cdots, M ; h, j=1, \cdots, M-d+1
$$

$\mathbf{T}_{i, s} \in R^{M-d+1}$ is a vector dependent on $s$ with the elements given by

$$
\mathbf{T}_{i, s}(h)=2 \cdot \mathbf{l}_{m+h+i-M}^{M-h+d+2}\left[\boldsymbol{\theta}_{s} \boldsymbol{\theta}_{s}^{T} \tilde{\boldsymbol{\varphi}}^{* T}(k+i)-\boldsymbol{\theta}_{s} y_{r}(k+i)\right]
$$

and $\mathbf{c}_{i, s}$ includes all terms independent of control signals. Matrices $\mathbf{S}, \mathbf{T}_{s}$ and $\mathbf{c}_{s}$ satisfy

$$
\mathbf{S}=\sum_{i=1}^{M} \mathbf{S}_{i}, \quad \mathbf{T}_{s}=\sum_{i=1}^{M} \mathbf{T}_{i, s}, \mathbf{c}_{s}=\sum_{i=1}^{M} \mathbf{c}_{i, s} .
$$

To minimize the loss $V_{M s}{ }^{\prime}$ with respect to $\mathbf{u}$, the following equation should hold:

$$
\frac{d V_{M s}^{\prime}}{d \mathbf{u}}=0
$$

From (25) and (29), the optimal control vector $\mathbf{u}_{s}^{m}$ is the solution of linear equation

$$
\mathbf{S u}_{s}^{m}=-\mathbf{T}_{s}
$$

At time $k$, only the last component of $\mathbf{u}_{s}^{m}$ is applied since the other components are future inputs.

Notet that $\mathbf{u}_{s}^{m}$ is the optimal control when $\boldsymbol{\theta}=\boldsymbol{\theta}_{s}$. Under the assumption (6), the suboptimal dual controller is the weighted sum of all possible $\mathbf{u}_{s}^{m}$ given by [16]

$$
\mathbf{u}^{m}(k)=\sum_{s=1}^{L} \mathbf{u}_{s}^{m}(k) \cdot q_{s}(k)
$$

The last component of $\mathbf{u}_{s}^{m}$ is then the obtained final control input. The proposed suboptimal dual control law is summarized as the following algorithm:

\section{Algorithm 1:}

Step 1. For all $s$, calculate matrices $\mathbf{A}_{s}, \mathbf{B}_{s}, \boldsymbol{\theta}_{s}$ in (12).

Step 2. Calculate matrix $\mathbf{S}$ from (26).

Step 3. Let $k=1$.

Step 4. Repeat

a. For all $s$, calculate $\mathbf{T}_{s}$ from (27) and (28) .

b. For all $s$, calculate optimal control $\mathbf{u}_{s}^{m}(k)$ from (30). 
c. Calculate the sub-optimal dual control $\mathbf{u}^{m}(k)$ from (31).

d. Update all parameters in Kalman filter according to (13) and (14).

e. Recursively calculate all posterior probabilities $q_{s}(k)$ according to (15).

f. Let $k=k+1$.

Step 5. End.

\section{Simulation}

In this section, the proposed controller (31) is tested in a simulation platform implemented in MATLAB/SIMULINK. The model of a real blood pressure control problem is studied in the simulation, which can be described by the difference equation (1) with input delay. In this model, the constants $m, n, M$ and the delay $d$ are chosen as $m=1, n=4, M=4, d=3$. The system parameters are assumed to belong to two sets, satisfying

$$
\boldsymbol{\theta} \in \Omega=\left\{\boldsymbol{\theta}_{1}, \boldsymbol{\theta}_{2}\right\}
$$

with

$$
\boldsymbol{\theta}_{1}=[-0.741,0.187,0,0,0.075]^{T}, \quad \boldsymbol{\theta}_{2}=[-0.865,0.149,0,0,0.09]^{T} .
$$

The variance of noise $\{e(k)\}$ satisfies $\sigma^{2}=0.001$. The initial condition of simulation is assumed to be

$$
q_{1}(0)=q_{2}(0)=0.5, y(0)=0.11, P_{s}(0 \mid 0)=10000 .
$$

The reference output is a slowly increasing slope followed by a constant signal. To verify the proposed controller, dual controllers without considering the uncertainties are also tested for comparison.

The tracking results using different controllers are shown in Figure 1. The system parameter vector is assumed to be $\boldsymbol{\theta}_{2}$. If the parameter vector are thought to be $\boldsymbol{\theta}_{1}$, there are significant tracking errors when using an optimal control of model 1 (see the blue broken line in Figure 1). If the proposed controller is used, the tracking result will quickly converge to the optimal result similar to that using an optimal control of model 2. The online calculated posterior probabilities are also recorded and plotted in Figure 2. It can be seen that the posterior probability of model $2, q_{2}(k)$, converges to 1 very quickly. This is also coincident with the tracking result of proposed controller. 


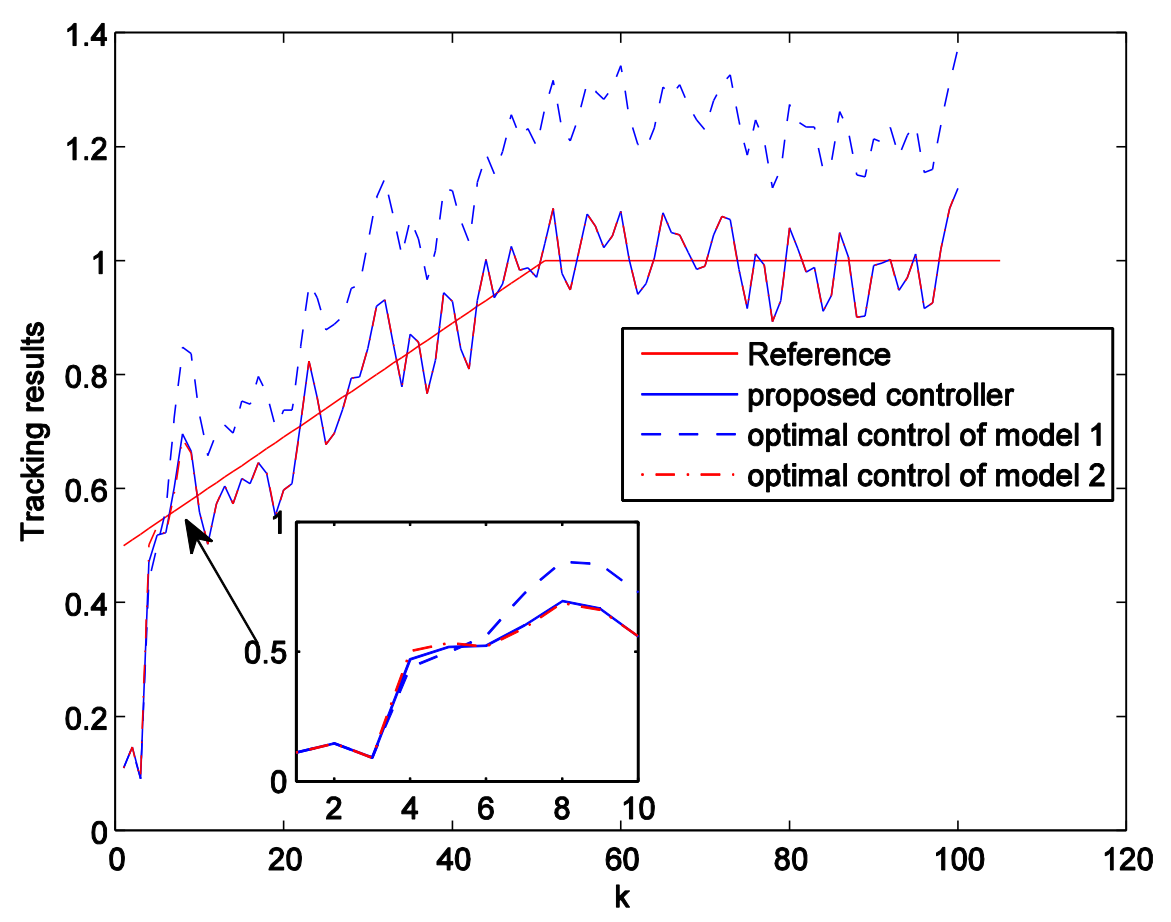

Figure 1. Tracking Results using Different Controllers

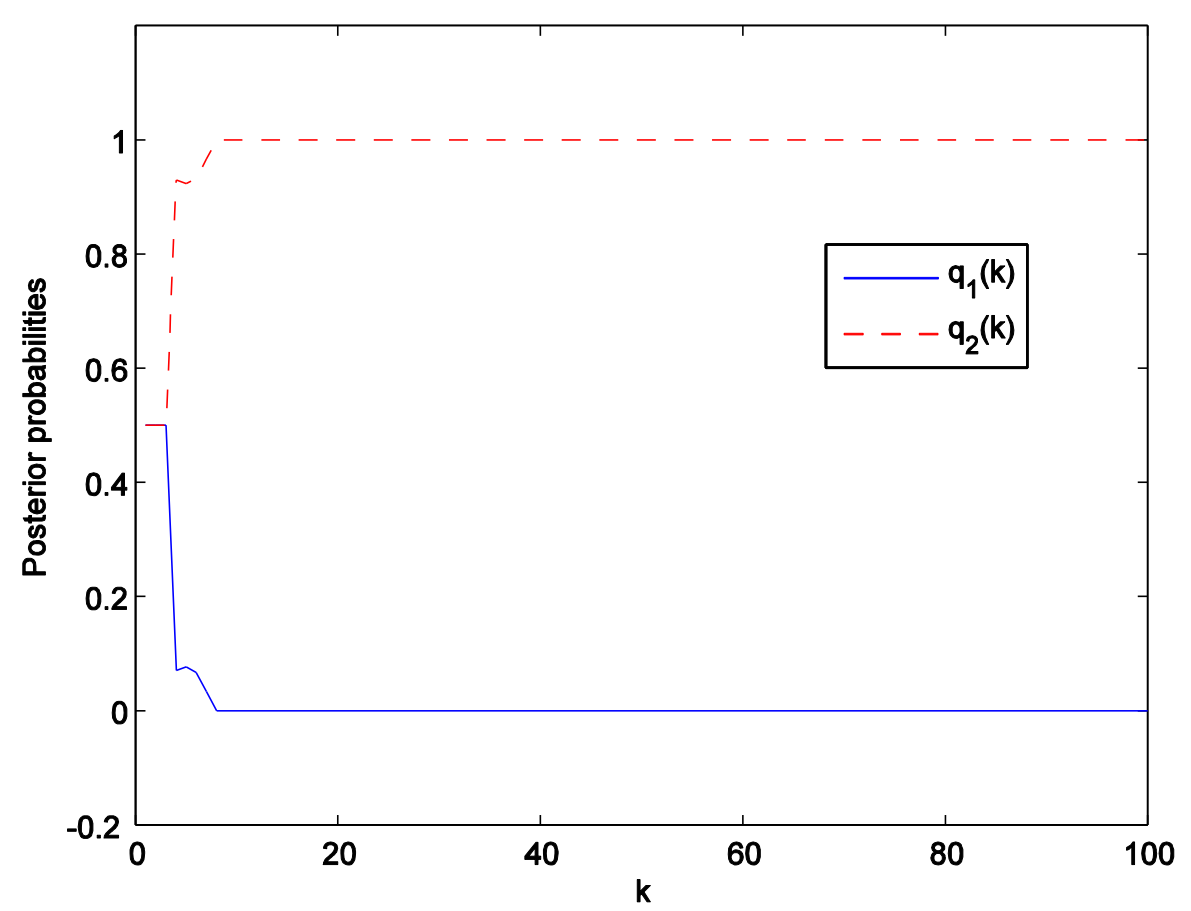

Figure 2. Posterior Probabilities at Time $K$. 


\section{Conclusion}

In this study, the dual control problem is discussed for the uncertain SISO discretetime system with input delay. The unknown system model parameters are assumed to be constant but belong to a known finite set. For each possible model, the Kalman filter is designed to online estimate the system state. It can also be used to calculate the posterior probability of model $s$. The final suboptimal dual controller is obtained as the weighted sum of optimal control for each possible model. A simulation platform is implemented in MATLAB/SIMULINK. A real blood pressure control problem is tested in the simulation platform by using different controllers. The simulation results verified the effectiveness of proposed dual controller.

In the future, we would like to investigate the dual control of the systems with timeinvariant input delay and extend current results to the multiple-in multiple-out (MIMO) cases.

\section{Acknowledgements}

The authors would like to thank the support by the Project 2110 of China.

\section{References}

[1] A. A. Fel'dbaum, Dual control theory I-II, Automation and Remote Control, vol. 21, (1960), pp. 874-880, 1033-1039.

[2] A. A. Fel'dbaum, Dual control theory III-IV, Automation and Remote Control, vol. 22, (1961), pp. 1-12, 109-121

[3] K. J. Astrom and B. Wittenmark, Adaptive Control, 2nd ed. Reading, MA: Addison-Wesley, (1995).

[4] B. Wittenmark, "Adaptive dual control methods: An overview", in 5th IFAC Symp. on Adaptive Systems in Control and Signal Processing, (1995), Jan, Budapest, Hungary, pp. 67-72.

[5] E. Tse, Y. Bar-Shalom, L. and Meier, Wide-sense adaptive dual control for nonlinear stochastic systems, IEEE Transaction on Automatic Control, (1973), Vol. 18, No. 2, pp. 98-108

[6] C. J. Wenk and Y. Bar-Shalom, "A multiple model adaptive dual control algorithm for stochastic system with unknown parameters", IEEE Transaction on Automatic Control, (1973), vol. 23, pp. 703-710.

[7] S. G. Fabri and V. Kadirkamanathan, "Functional Adaptive Control: An Intelligent Systems Approach. London", UK: Springer-Verlag, (2001).

[8] N. M. Filatov and H. Unbehauen, "Adaptive Dual Control: Theory and Applications", London, UK: Springer-Verlag, (2004).

[9] R. Milito, C. S. Padilla, R. A. Padilla and D. Cadorin, "An innovations approach to dual control", IEEE Transactions on Automatic Control, vol. 27, (1982), pp. 133-137

[10] A. L. Maitelli and T. Yoneyama, "A two-stage suboptimal dual controller for systems with stochastic parameters using optimal predictors", IEE Proc.-Control Theory and Application, (1994), vol. 11, pp. 225260

[11] D. Li, F. C. Qian and P. L. Fu, "Variance minimization approach for a class of dual control problems", IEEE Transactions on Automatic Control, vol. 47, (2002), pp. 2010-2020.

[12] P. L. Fu, D. Li and F. C. Qian, "Active dual control for linear quadratic Gaussian system with unknown parameters", in: Proc of the 15th IFAC World Congress, Barcelona, Spain, (2002).

[13] C. Ramesh, H. Sandberg, L. Bao and K. H. Johansson, "On the Dual Effect in State-Based Scheduling of Networked Control Systems", in: Proc 2011 American Control Conference on O'Farrell Street, San Francisco, CA, USA, (2011).

[14] A. L. Maitelli and T. Yoneyama, "Suboptimal Dual Adaptive Control for Blood Pressure Manage-

Ment”, IEEE Transactions on Biomedical Engineering, vol. 44, pp. 486-492, (1997).

[15] F. Casiello and K. A. Loparo, "Optimal policies for passive learning controllers", Automatica, vol. 25, (1989), pp. 757-763.

[16] J.G. Deshpande, T.N. Upadhyay and D.G. Lainiotis, "Adaptive control of linear stochastic systems", Automatica, vol. 9, (1973), pp. 107-115. 


\section{Authors}

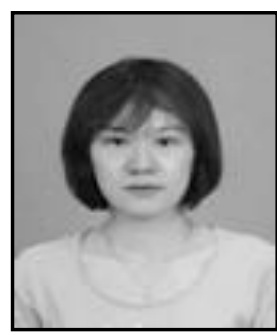

Suping Cao, she was born in Anhui, China. She received her BS and MS degrees from the Second Artillery Engineering University, China in 2001 and 2004, respectively. She is currently pursuing her $\mathrm{PhD}$ in the School of Automation, Huazhong University of Science and Technology, China. Her main research interests include the stochastic adaptive control, pattern recognition and small target detection and tracking.

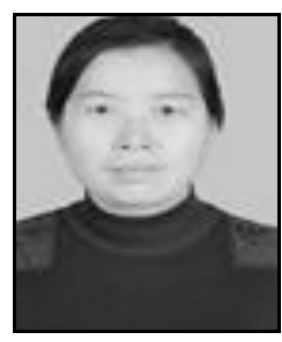

Xizhen Hu, she was born in HuBei, China. She received her MS and $\mathrm{PhD}$ degrees from WuHan University, China in 1998 and 2004, respectively. Her main research interests include the system optimization, the traffic optimization modeling, and optimum control. 
International Journal of Control and Automation Vol. 9, No. 7 (2016) 